P148 (continued)

estimate adjusted odds ratios (AOR) for consuming SSBs $\geq 3$ times/day based on knowledge of SSB-related health conditions using $<1$ time/day as the reference category.

Results: Overall, 36\% of Hispanic adults consumed SSBs $\geq 3$ times/day. Although most identified that weight gain $(75 \%)$ and diabetes $(76 \%)$ are related to drinking SSBs, only half identified that cavities $(57 \%)$ and hypertension (41\%) are related to drinking SSBs. Even fewer adults identified high cholesterol (32\%) and heart disease (32\%) as related to drinking SSBs. In crude analyses, lower SSB intake was associated with knowledge of the relationships between SSBs and weight gain, cavities, and heart disease $\left(\mathrm{p}<0.05, \chi^{2}\right.$ test); however, after adjusting for age, sex, education, marital status, income, weight status, region, country of origin, and acculturation, these associations were no longer significant.

Conclusions and Implications: Most Hispanic adults knew that SSB intake was related to weight gain and diabetes, but knowledge was limited for other health conditions. Knowledge of SSB-related health conditions alone may not be related to reported SSB intake, and therefore insufficient for Hispanic adult behavior change.

Funding: None

\section{P149 Mobile Apps for the Dietary Approaches to Stop Hypertension (DASH): App Quality Evaluation}

Kristen DiFilippo, MS, LDN, RD, kdifilip@illinois.edu, University of Illinois, 238 Bevier Hall, 905 South Goodwin, Urbana, IL 61801; Karen Chapman-

Novakofski, PhD, LDN, RD, University of Illinois

Objective: Considering potential for supporting behavior change, DASH app quality was evaluated.

Study Design, Setting, Participants, Intervention: DASH apps were identified (App Store); ranked daily for 1 month, producing 5 top unique free apps and 1 paid app. Searching all DASH apps manually identified four more. Six were excluded: 1 recipe app, 1 required additional equipment, 1 had limited DASH content, 3 included other diets. Dietitians $(n=5)$ who used apps at work and with $>1$ year cardiovascular experience evaluated free apps, 3 also evaluated the paid app, using the previously validated app quality evaluation (AQEL). The female, 31.8 (25-42) year olds had 5.2 (2-12) years' experience (varied settings).

Outcome Measures and Analysis: Two-way random, absolute intra-class correlations, average measures (ICC) assessed inter-rater reliability. AQEL rates 7 domains 0-10: Behavior Change Potential, Function, Adult Appropriateness, Knowledge, Skill, DASH Relevance, and Purpose.

Results: Free app's $\operatorname{ICC}(7,5)>.7$, (95\%CI .4 to .95); paid app ICC $(7,3)=.2$ (95\%CI -.07 to .67). Removing 1 low rater improved paid app AQEL reliability, $\operatorname{ICC}(7,2)=.95$ (95\%CI .65 to .98); 2 free apps improved slightly. AQEL ratings: Behavior all $\leq 6$; Function and Adult Appropriateness all $\geq 7$; Knowledge all free apps $<6$, paid 7.4 (9.1 with rater removed); Skill all free $<6$, paid 8.5 (10 with rater removed); DASH relevance all free $<6.5$, paid 7.1 (10 with rater removed); Purpose 6.3 for 1 free app, $\geq 8$ all other apps.

Conclusions and Implications: DASH apps that function well technically are available; other quality domains are low in free DASH apps and moderate in knowledge/skill for paid app. Additional DASH-related development to support behavior change seems warranted.

Funding: None

\section{P150 WITHDRAWN}

